\title{
Acceso a la justicia de sectores especialmente vulnerables en el Programa Nacional de Casas de Justicia y Paz
}

Línea de investigación: Derecho y sociedad

Director de la Investigación: Profesor Luis Alfonso Fajardo

\section{PLANTEAMIENTO DEL PROBLEMA}

\subsection{El acceso a la justicia en Colombia}

Durante las últimas décadas, en Colombia el sistema de administración de justicia ha sufrido un proceso de deslegitimación creciente generado por distintos factores, como la congestión, el retardo en la resolución de los asuntos, el exceso de procesalismo, los fenómenos de corrupción, las presiones externas, etc. lo cual viene generando, como consecuencia, formas de injusticia y exclusión. Las comunidades más vulnerables de la población colombiana constantemente se encuentran frente a la violación de sus derechos fundamentales y garantías constitucionales, sin los medios para poder acudir al apoyo del Estado, el cual debería ser garante de aquéllos. La Constitución Política, en su art. 229, "garantiza el derecho de toda persona para acceder a la administración de justicia". En desarrollo de este derecho, distintos gobiernos han impulsado el Programa Nacional de Casas de Justicia, establecimientos creados para el servicio precisamente de aquellos sectores que, por sus condiciones sociales, económicas o culturales, tienen un acceso restringido al aparato de justicia estatal.

La estructura normativa del moderno derecho positivo formal es poco eficaz y no consigue atender las necesidades de las actuales sociedades periféricas, como la colombiana, aquejadas de una profunda crisis de legitimidad y de funcionamiento de la justicia. La nueva propuesta de legalidad se basa en un cierto tipo particular de pluralismo capaz de reconocer normas extra e infraestatales, originadas por las 
carencias y las necesidades insatisfechas, provenientes de los nuevos actores sociales, afirmándose un tipo de pluralidad jurídica abierta, flexible, participativa y democrática que contenga todos los intereses cotidianos individuales y colectivos.

Los presupuestos sustantivos que constituyen y sustentan nuevas formas de legitimación requieren de la justicia del derecho y deben ser buscados en la acción participativa de los sujetos sociales que emergen y en la justa satisfacción de sus necesidades fundamentales, lo que significa adoptar estrategias de acción vinculadas a la participación consciente y activa, capaz de actuar de forma solidaria y emancipadora.

El Estado ahora le apuesta al incremento de recursos en la gestión de conflictos, ampliando el mercado y, por lo tanto, la oferta con tal fin, contando con dos formas para hacerlo:

- Justicia participativa. Ésta se entiende de dos maneras: la primera, generando un cambio para gestionar y direccionar el tratamiento de un conflicto mediante la ampliación de gestión de los sujetos $y$, una segunda, mediante la creación de un escenario en donde la producción del derecho sea una forma de transformar la sociedad.

- Justicia alternativa. Ésta debe contar con unos instrumentos distintos a los de la justicia estatal, en el cual se produce una alternatividad al orden jurídico político existente, generando un nuevo orden de regencia y de participación que pretende ser más democrático e incluyente.

Con el aumento de los niveles de pobreza, la prolongación del conflicto interno, el desplazamiento, etc., la población vulnerable se ha incrementado y, con ello, sus carencias y necesidades. Cada vez son más los colombianos que se encuentran en los cinturones de miseria de las ciudades, que buscan una salida rápida y efectiva a sus problemáticas sociales. En esta carrera en contra de la injusticia social, amplios sectores de la población continúan excluidos. Básicamente, estos sectores estan conformados por recicladores, trabajadores sexuales, pueblos indígenas, afrocolombianos y comunidades desplazadas, entre otros, que no ven una solución concreta y efectiva a las dificultades que encuentra en el diario vivir.

Cada uno de estos grupos poblacionales, en su calidad de ciudadanos colombianos, posee unas garantías representadas en derechos, entre ellos el acceso a la prestación de los servicios públicos, siendo uno de ellos el sistema judicial. El acceso a la administración de justicia que dicho sistema debería garantizar se ve limitado fundamentalmente por tres factores: Geográfico, que se refiere a las limitaciones especiales que aquejan a muchas comunidades para acceder al servicio de justicia; económico debido a los costos de los procesos (civiles, penales, laborales, etc.), dejando a muchos sectores de la población vulnerable sin capacidad para acudir ante las instancias judiciales; por último, el factor cultural, que se refiere al desconocimiento de la ley y de sus procedimientos. Amén de ello la falta de identificación con la normatividad vigente, que plantea el problema de legitimidades.

No se puede desconocer que el Estado colombiano en los últimos años ha tratado de dar solución a esta problemática. Sin embargo, los actores que se encuentran continuamente vulnerados en sus derechos no reconocen los cambios o apenas perciben si los reciben. Es necesario, por lo tanto, que los instrumentos creados para solucionar el problema sean eficaces $y$, para esto, los medios son importantes, así como la participación de la comunidad en la funcionalidad de los mismos. 
El programa Nacional de Casas de Justicia y Paz, del Ministerio del Interior y de Justicia, fue creado con el fin de facilitar el acceso a la justicia formal y no formal de las comunidades más vulnerables para lograr la resolución de los conflictos y el fortalecimiento de la convivencia pacífica. En 1994, bajo el Gobierno del presidente Ernesto Samper, se firmó un convenio interinstitucional entre el Ministerio de Justicia (en ese entonces), la Fiscalía, Medicina Legal, ICBF, FES, Registraduría Nacional y Procuraduría, que le dio vida al Programa Nacional de Casas de Justicia. Posteriormente, en el 2000, el proceso se fortalece e institucionaliza definitivamente con la expedición del Decreto 1477 que adopta el Programa Nacional de Casas de Justicia, prioritariamente en las zonas marginales, en las cabeceras municipales y en centros poblados de los corregimientos. Dicho Programa nace como desarrollo de los artículos 229 y 113 de la Constitución Nacional, y el art. 2 de la ley 270 de 1996, referentes a la garantía de acceso a la justicia de todos los ciudadanos colombianos.

Las Casas de Justicia son centros multiagenciales de información, orientación, referencia y prestación de servicios de resolución de conflictos, donde se aplican y ejecutan mecanismos de justicia formal y no formal. En ellas se busca que las personas sean atendidas de manera eficaz y gratuita, dando respuesta centralizada a sus inquietudes mediante la acción coordinada y solidaria de sus funcionarios.

Este programa tiene como objetivos:

- Ampliar la cobertura de la administración de justicia.

- Ofrecer a la ciudadanía espacios integrales de acción en justicia.
- Formar a la comunidad y a los funcionarios de estos centros en el manejo de la metodología para el uso y la difusión de los Mecanismos Alternativos de Solución de Conflictos -MASC-.

- Propiciar la participación efectiva de la comunidad en el diagnóstico y solución de conflictos mediante mecanismos formales y no formales.

- Establecer espacios de participación y pedagogía ciudadana que contribuyan a la construcción de una conviviencia pacífica.

- Articular los políticas de justicia con los programas de desarrollo comunitario ${ }^{1}$.

Una de las características de las Casas de Justicia es que se encuentran conformadas por instituciones de distinta naturaleza que prestan una gran variedad de servicios a la comunidad. De ahí, su carácter multiagencial y la importancia que tiene el trabajo coordinado que articula acciones y recursos humanos, técnicos y físicos. Algunas de las instituciones que se encuentran presentes en las Casas de Justicia son Fiscalía, Secretaría de Gobierno Municipal, Bienestar Familiar, Defensoría, Registraduría, Personería, Medicina Legal, Universidades, Justicia de Paz, centros de conciliación, conciliadores en equidad y la alcaldía.

Las Casas de Justicia han contribuido al fortalecimiento de la presencia estatal en sectores del territorio nacional en donde era bastante limitada. Así mismo, han permitido articular las políticas de justicia con el desarrollo social y comunitario. La defensa de los Derechos Humanos es una de sus banderas, permitiendo desarrollar capacitación sobre este tema en las comunidades, ampliando las perspectivas de desarrollo y su- 
pervivencia de las mismas, al procurar mejorar la calidad de vida de los moradores de su área de influencia.

Las primeras experiencias de Casas de Justicia y Paz se originaron en 1995, cuando se creó la Casa de Justicia de Ciudad Bolívar con ayuda de la alcaldía municipal; a la par, se construyó la Casa de Justicia en el sector de Aguablanca, en Cali. La presencia de estos centros se ha extendido por todo el país, buscando ampliar la cobertura en la prestación del servicio. Se cuenta con Casas de Justicia en la mayoría de regiones que conforman el territorio nacional, distribuidas en los departamentos y localizadas en los centros urbanos de mayor afluencia de población, entre ellos: Bogotá, Cali, Valledupar, Ibagué, Bucaramanga, Pereira, Chigorodó, Barranquilla, Neiva, Cartagena, Popayán, Pasto, Medellín, Riohacha, San Andrés, Mocoa, Puerto Asís, Manizales, Villavicencio, Armenia, Bello, Girardot, Tuluá, Cartago, Floridablanca, Santa Marta, Cúcuta, Soacha, Yopal, Quibdó, Buga y Chía.

Las Casas se establecen en zonas marginales de las grandes ciudades del país, aun cuando no niegan su servicio a ningún ciudadano. Su radio de influencia directa abarca los barrios, zonas y comunas cercanas donde habitan la mayoría de sus usuarios. En estos sectores, las condiciones de vida son generalmente difíciles: el acceso a los servicios públicos y la calidad de la educación y de los servicios de salud no satisfacen las necesidades de toda la población; en muchos de los casos, estas zonas de las grandes ciudades cuentan con un número considerable de barrios subnormales y de asentamientos de familias en situación de desplazamiento. Los niveles de conflictividad son altos, primando problemáticas como la violencia intrafamiliar, el abuso sexual de niños y niñas, el abandono de la juventud que se refleja en altos índices de drogadicción, prostitución, pandillas y delincuencia común, la manera violenta de resolver las diferencias en- tre vecinos, la existencia de bandas dedicadas al hurto y las lesiones personales. Además, en estos escenarios también se evidencia la presencia de grupos armados ilegales, el reclutamiento forzoso de jóvenes el narcotráfico. Todas estas circunstancias perturban la vida cotidiana y dificultan la existencia de normas colectivas y valores que propicien y salvaguarden la convivencia pacífica.

Las Casas de Justicia se han instaurado en estos sectores de la población para remediar la problemática que se presenta y dar respuesta a las necesidades básicas de sus habitantes, como institución garante de la presencia del Estado y el acceso a la justicia; igualmente como promotoras de la convivencia pacífica, de los Derechos Humanos y de Ios MASC que faciliten la participación de la comunidad.

El programa ha implementado un sistema para recolección de información con el fin de elaborar los planes de acción en cuanto a la prestación del servicio, para lo cual instituyó el Censo Nacional de Casas de Justicia, que busca conocer el número de las demandas y sus principales características y tendencias. Este censo se viene realizando desde 2001, mostrando tendencias como la mayor afluencia de las mujeres a estos centros de resolución de conflictos, con el $60.2 \%$ de la demanda, y la poca receptividad de este medio por parte de los jóvenes y adultos mayores. Así mismo, se destaca el número significativo de desempleados que acuden. El mayor índice de conflictividad está representado por conflictos de índole familiar con un $46.8 \%$. El porcentaje restante se refiere a conflictos generados por pérdida de documentos, asuntos penales menores y conflictos entre vecinos.

Los indicadores nos muestran unos resultados importantes acerca de la labor desempeñada por las Casas de Justicia en el país, al demostrar que cada vez es mayor el número de personas que acuden a ellas y las problemáticas atendidas y 
resueltas. Pero también observamos que no se hace referencia a los avances en cuanto a los programas especiales para grupos minoritarios y/o más vulnerables, como las comunidades desplazadas o grupos étnicos, entre otros. En el consolidado nacional se observa que tan sólo el 1.0\% del motivo de las consultas fue por problemas relacionados por el desplazamiento forzado. No se hace referencia tampoco o no existe otro indicador que muestre de forma clara y precisa resultados en cuanto a otros grupos sociales vulnerables.

Si bien es cierto se deja claro que "la comunidad atendida en las Casas no es susceptible de ser interpretada como reflexión de la realidad social de la zona ni comparada con la potencial demanda de la población victimizada objeto de las Casas, puesto que se carece de la información suficiente para establecer dichas relaciones $^{\prime 2}$, esta afirmación nos demuestra que la cobertura de estos centros sigue siendo limitada, lo cual no debe ser un freno para posibilitar la ampliación de sus campos de acción.

Los sectores de la población más vulnerable constituyen los principales actores hacia los cuales se concentra la labor que desempeña esta clase de programas. Esta dificultad se podría sortear permitiendo la intervención más activa de la comunidad, en la formulación de los planes de acción propuestos, que permita ampliar la cobertura de dichas instituciones hacia problemáticas que parecen han sido poco tratadas hasta el momento, como las concernientes a conflictos generados por el desplazamiento, los riesgos y problemas generados a raíz del trabajo desempeñado (trabajadores sexuales, recicla-dores, vendedores ambulantes, mendigos), o el grupo étnico al que se pertenece. Creemos que la información y la comunicación en una doble vía con la comunidad es un factor determinante para el desarrollo efectivo del programa de Casas de Justicia.

\subsection{Formulación del problema}

La investigación intenta resolver la pregunta:

¿Están dando respuestas las Casas de Justicia a los reclamos de acceso a la justicia de los sectores más vulnerables del país, como son pueblos indígenas, comunidades afrocolombianas, grupos de desplazados por la violencia, adultos mayores, trabajadores sexuales, habitantes de la calle y vendedores ambulantes?

\section{OBJETIVOS}

\subsection{Objetivos general}

Evaluar las formas de acceso a la justicia ofrecidas desde las Casas de Justicia y Paz a grupos de población especialmente vulnerables: pueblos indígenas, comunidades afrocolombianas, desplazados por la violencia, adultos mayores, trabajadoras/es sexuales, habitantes de la calle y vendedores ambulantes.

\subsection{Objetivos específicos}

- Estudiar las dificultades u obstáculos y los niveles de eficiencia de las formas de acceso a la justicia a estos sectores.

- Elaborar un protocolo de atención para garantizar el acceso a la justicia de estos sectores especialmente vulnerables.

- Preparar informes sobre la situación de las Casas de Justicia respecto a estos sectores vulnerables. 


\section{MARCO TEÓRICO}

\subsection{Estado del arte}

En términos generales, se considera que parte fundamental del derecho alternativo es la creación de grupos de apoyo jurídico popular que buscan defender intereses colectivos mediante la organización comunitaria y la capacitación legal orientada hacia la movilización y la organización ${ }^{3}$. Desde este punto de vista, las Casas de Justicia se orientan, hacia esta perspectiva del derecho. En su conformación y objetivos, se piensa una nueva forma de asumir los servicios legales, de tal manera que responda en forma real a los problemas que afronta la sociedad colombiana, en especial los sectores más vulnerables y que por diversas circunstancias tienen un acceso restringido a un servicio legal eficiente, seguro y al alcance de sus posibilidades en términos económicos. Se debe tener en cuenta que los objetivos se orientan hacia un uso alternativo del derecho; pero al indagar en los indicadores de gestión que muestra el Censo Nacional de Casas de Justicia, pareciera que el objetivo se difumina y pierde de vista el impulso real para Ios MASC o el desarrollo de las formas dejusticia comunitaria.

Al respecto, el teórico Manuel Yaques, abogado chileno, nos expone la perspectiva del uso alternativo del derecho, planteando que es necesario "imaginar estrategias jurídicas que planteen un uso alternativo del derecho, que cuestione de fondo el papel tradicional asignado al ejercicio de la profesión y la manera como ésta se relaciona con la comunidad, permitiendo revisar los contenidos y los métodos clásicos del traba- jo legal, introduciendo elementos nuevos que le otorguen vida y vitalidad al derecho como componente dinámico de la opción de cambio"4

Así, al socializar el conocimiento del derecho y posibilitar la construcción normativa desde la comunidad, se logra una acercamiento real a la problemática social, respondiendo a las necesidades específicas de cada grupo. La construcción del derecho desde el interior de los grupos sociales permitirá tener conocimiento $\mathrm{y}$, por lo tanto, acceso al mismo.

Partiendo de las coyunturas que vive la sociedad latinoamericana, y del caso concreto colombiano, se ha visto la necesidad de repensar las estructuras políticas y organizacionales para ser replanteadas desde el ámbito comunitario, estableciendo nuevas estrategias de desarrollo y cambio que configuren una base real para la democracia y la participación ciudadana. El punto de partida es la insatisfacción de necesidades frente a las cuales el Estado está en la obligación de dar una respuesta inmediata y satisfactoria.

El tema ha tenido, sin duda, aproximaciones diversas: "reorientar el desarrollo no al crecimiento económico, sino a la satisfacción de necesidades del hombre; que dichas necesidades no son satisfechas por el sistema vigente; que la forma de satisfacción de las mismas se ha de hacer con la participación de los diversos sectores sociales y no por una clase privilegiada y excluyente; la importancia que tiene el movimiento popular en la estructuración de las opciones políticas" ${ }^{5}$.

Partiendo del marco de referencia elaborado por Manuel Yaques, sobre los criterios establecidos para identificar un servicio legal alternativo, he-

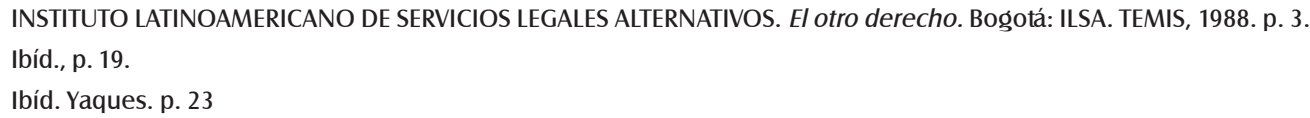


mos establecido que el Plan Nacional de Casas de Justicia y Paz, en un principio, podría responder a la tipología de los servicios legales alternativos. Como primera medida, tenemos que el área de atención a la que se orienta dicho servicio se ubica en los sectores de la población que por sus características socio-culturales se encuentran dentro de los sectores más vulnerables, utilizando como método de trabajo una estructura interdisciplinaria, incorporando estrategias de defensa legal que sugieren el rígido sistema jurídico nacional, en teoría. Si nos acercamos y vemos más profundamente el funcionamiento de las Casas de Justicia, se puede reconocer que la superación de dichas formas rígidas aún no se logra del todo.

Dentro de las rutas de solución de conflictos, se tiene que tan solo un $0.2 \%$ se lleva a cabo por intermedio de la conciliación en equidad o los jueces de paz, siendo las estructuras del rígido sistema estatal de justicia, como la Fiscalía General de la Nación, las que atienden el mayor número de demandas en un $31.8 \%$. Es preciso señalar que las Casas de Justicia son creadas desde la institucionalidad estatal y, como tales, pueden seguir reproduciendo sus vicios. Por lo tanto, es necesario impulsar dentro del programa, el objetivo real del mismo para que no se diluya y pierda en la maraña de formas legales tradicionales.

Al hacer referencia a un servicio legal transformador, creemos que el servicio de Casas de Justicia, en su corta trayectoria, no ha dado respuesta al cambio real, pero sí ha dejado la puerta abierta para ser un instrumento del mismo. En este punto, se pretende que la clase de servicio creado oriente su trabajo hacia la resolución y cumplimiento de la satisfacción de las necesidades de la comunidad, posibilitando un cambio en las estructura sociales. Los indicadores nos mues- tran que dicho servicio ha respondido en buena medida a las problemáticas que se le presentan, pero éstas no corresponden a la totalidad de necesidades insatisfechas de los sectores más vulnerables. El censo realizado nos muestra que el mayor número de conflictividades resueltas se refiere a conflictos intrafamiliares y problemas entre vecinos. Si bien la resolución de este tipo de conflictos incide en el buen desarrollo comunitario, no son un factor real de cambio de la realidad social que se vive, haciendo necesario que el total de las conflictividades que se presentan acceda a su resolución a través de las Casas de Justicia.

Problemáticas originadas por el desplazamiento -uno de los grandes problemas sociales que aqueja a la sociedad colombiana- son tratadas en niveles mínimos por estos organismos (tan sólo el 1\% de las demandas de las Casas de Justicia son de esta índole $)^{6}$. Se debe tener en cuenta que, en la gran mayoría de los casos, el área de influencia de las Casas de Justicia se ejerce en sectores de los centros urbanos deprimidos, donde el mayor número de la población es desplazada. Como ejemplo, podemos citar las Casas de los municipios de Quibdó, Buga, Yopal y Soacha. El programa de Casas de Justicia, reconociendo la crítica situación de la población desplazada, ha instaurado dentro de sus planes de acción orientaciones para la atención a esta población, pero dichos planes no han tenido una eficaz recepción por parte la comunidad usuaria de las Casas, lo cual se muestra en las estadísticas dadas en el censo. Se hace necesario plantear estrategias para que los programas orientados hacia este tipo de conflictividades se concreten en resultados favorables para los usuarios.

Para el acceso a la administración de justicia estatal, se han desarrollado estrategias muy diver-

${ }_{6} \quad$ MINISTERIO DEL INTERIOR Y DE JUSTICIA. Casas de Justicia .... Op. cit. p. 39. 
sas, que van desde la reorganización del aparato judicial hasta el impulso de programas de educación popular legal, pasando por programas de asistencia jurídica, desde los más diversos ribetes ideológicos. Sin embargo, en la medida que crece el desfase entre los procesos de judicialización y juridización, tales estrategias tienden a quedarse cortas porque no atienden de fondo el desequilibrio que hay entre una y otra (Ardila: 88).

El problema del acceso a la administración de justicia es, en el fondo, el de la garantía efectiva de los derechos establecidos en el orden jurídico. Sin acceso a su tutela, mediante el sistema de administración de justicia, el aparato que ofrece la estructura judicial estatal a determinados intereses resulta nugatorio. Lo cual está en la escena de la ciudadanía (Ardila: 87)

La metodología empleada es la manera como el programa legal se relaciona con el beneficiario, introduciendo métodos de capacitación y participación de la propia comunidad en la solución de su propio conflicto. Las Casas de Justicia y Paz de Colombia dentro de sus planes de acción han establecido programas de capacitación de la comunidad, donde el objetivo es instruir a los miembros de la comunidad sobre temas como derechos humanos, democracia y justicia, luego de debatidos estos temas en talleres, en donde participa el común de la comunidad, se elaboran unos módulos que recogen las experiencias y percepciones de la comunidad respecto a los temas tratados. De estos módulos tenemos referencia de cuatro de ellos, aunque conocemos de la existencia de más ejemplares: Módulo 1 "Derechos Humanos y vida cotidiana» (Casa de Justicia Barranquilla) y Módulo 2 «Democracia, justicia y convivencia» (Casa de Justicia de Barranquilla) y sus homólogos desarrollados y publicados por la Casa de Justicia de Ciudad Bolívar, en Bogotá.

En los Planes de Acción del Programa de Casas de Justicia ${ }^{8}$ sobresalen los siguientes aspectos:

- Violencia intrafamiliar: hay diversos enfoques que van desde cómo mejorar la intervención de manera integral hasta el énfasis en temas de abuso sexual y prostitución infantil, así como actividades de prevención con alto contenido de participación comunitaria y de acercamiento coordinado a redes se solidaridad.

- Fomento a Ios MASC: se reivindican las tareas de formación y promoción, pero con marcado interés por incidir directamente en el tratamiento de problemáticas identificadas como prioritarias en las comunidades del entorno de las Casas, en las cuales sobresalen también la violencia intrafamiliar y el tema de los jóvenes.

- Asuntos étnicos: el tema étnico aparece en aquellas Casas de Justicia cuyos contextos poblacionales tienen presencia significativa de comunidades étnicas. En este sentido, las cuatro etnias minoritarias reconocidas como tales en el país son sujeto importante para mejorar la oferta de servicios por parte de las Casas y fundamentalmente para fomentar el reconocimiento de sus jurisdicciones o, en otros casos, el respeto a sus tradiciones culturales sobre el tratamiento de los conflictos dentro de sus comunidades.

ARDILA, Édgar. Variaciones sobre la justicia comunitaria: claves para el estudio de las políticas de justicia comunitaria. Bogotá: ILSA. 2004.

$8 \quad$ PROGRAMA NACIONAL DEL CASAS DE JUSTICIA. Señales de convivencia, n. 7. Bogotá, 2004. p.3. 
- Jóvenes: los planes de acción formulados ponen en evidencia la carencia de oportunidades para los jóvenes y el alto riesgo de agudización de dinámicas de conflictividad. Las Casas que abordan este tema reconocen la necesidad de diseñar estrategias y metodologías propias de acercamiento e intervención con este sector de la población en cuanto al tema de acceso a la justicia y convivencia pacífica.

- Educación legal: si bien este tema no aparece como enunciado como tal en ninguno de los planes de acción, son varias las Casas que introdujeron en su plan de acción objetivos y actividades relacionadas con la necesidad de promover espacios, escenarios y mecanismos de formación y participación comunitaria, que potencien la capacidad ciudadana para acceder a los servicios de administración de justicia y para ejercer plenamente sus derechos individuales y colectivos.

- Convivencia: se destaca en este tema la estrategia con que contaron las Casas para activar, desde el momento mismo de su establecimiento, la participación y el desarrollo comunitario, trabajo que logró articular, alrededor de planes de convivencia pacífica, los imaginarios y las reivindicaciones de la comunidad, quedando consignados en los planes de acción.

- Derechos Humanos. Éste es un objetivo importante del programa, dada la estrecha relación con el tema de acceso a la justicia. Muchas Casas de Justicia privilegiaron en sus estrategias y objetivos la promoción de los Derechos Humanos, planteando una conexión estrecha con el tema de prevención, según la tipología de conflictos más demandados.
- Desarrollo institucional. Éste aspecto denota un alto nivel de preocupación e interés de las Casas por subsanar las dificultades que entorpecen el trabajo cotidiano y, así mismo, por potenciar las fortalezas que han redundado en la generación de confianza en las comunidades. Sobresalen temas como la operatividad de los convenios interinstitucionales, mejoramiento de la presencia institucional y la permanencia institucional, en busca de formas que garanticen una mayor atención integral en el servicio prestado.

Un servicio legal alternativo es un mayor grado transformador en la medida que se oriente no sólo a la prestación de un servicio, sino también que se inscribe en una perspectiva de estrategia de cambio de las estructuras sociales (Yaques: 35 ).

En cuanto a la acción temporal de los servicios legales alternativos, se distinguen dos tipologías:

- Servicio alternativo coyuntural: será aquel que cuando habiendo cumplido todas las exigencias y requisitos pretendidos para un servicio legal innovador se extingue por modificarse la coyuntura y las circunstancias que lo hicieron funcionar. Se excluyen las apreciaciones de orden financiero.

- Servicio legal alternativo permanente: define su estrategia y naturaleza en una perspectiva constante de cambio de las estructuras sociales económicas, políticas y jurídicas dominantes (Yaques: 37).

Se habla de crisis del derecho en tanto se reduce la eficacia de la norma legal como orientadora de las relaciones sociales, y se habla de crisis de la administración de Justicia en tanto la capacidad del aparato judicial para atender la 
conflictividad es desbordada ${ }^{9}$. En cuanto a la judicatura, la crisis se manifiesta en su insuficiencia estructural para atender toda la conflictividad: se congestionan los tribunales, los procesos se hacen cada vez más lentos y la impunidad se incrementa. En cuanto al sistema jurídico, la crisis se manifiesta en la insuficiencia del derecho para resolver muchos de los conflictos. Es lo que se ha denominado la "crisis del paradigma de la legalidad estatal monista" (Wolkmer, 1994: 91). Debido a esa crisis, muchos de los asuntos tienden a ser tratados por el régimen como asuntos que se entran a considerar extrajurídicos ${ }^{10}$. La ilegitimidad del aparato judicial y el sistema jurídico estatal se manifiesta en una creciente tendencia al uso de mecanismos ajenos a ellos para el manejo de la conflictividad. En esas condiciones, reducir la administración de justicia al aparato judicial estatal y el derecho al del Estado en esta época sería ir en contra de las evidencias y ya no se podría sostener con el más mínimo asomo de realidad (Ardila: 8$)^{11}$.

Efectivamente se puede practicar el derecho, entendido como derecho formal o estatal del manera alternativa, es decir intentando hacerlo receptivo a las expectativas de quienes tienden a menudo a ignorar, e incluso utilizarlo para fomentar determinadas luchas emancipatorias (Eberhard: 16) ${ }^{12}$. Es un hecho que el Estado de derecho modernos necesita de una infraestructura pesada y costosa; por lo tanto, no puede administrar a toda la sociedad desde un monopolio, es necesario que algunos poderes sean delegados, que se encuentren articulaciones entre los diversos actores sociales. De esta ma- nera, se ponen en juego lógicas institucionales más funcionales que se expresan en escenarios diversos que pueden ser tradicionales o modernos, de negociación o confrontación, en función de lo que la parte ponga en juego y que se materializarían en unos mandatos negociados, aceptados o impuestos (Eberhard: 27).

La experiencia ha demostrado que a menudo la puesta en marcha de lo informal en el Estado ha resultado, por una parte, más en un control mayor del Estado sobre las diversas colectividades que en la garantía de una cierta autonomía para éstas. Como lo señala Richar Abel, citado por Eberhard:

Si las instituciones formales permiten a los desfavorecidos tener más fácilmente acceso a la justicia, puede ser todavía más significativo que ellas permitan a la administración pública tener acceso a los desfavorecidos.

Y Fernando Rojas (1992: 426 - 427) señala que:

Los servicios jurídicos, especialmente los nuevos, tienen tendencia a canalizar todo conflicto a través del sistema jurídico, a reforzar la sumisión de la clase subordinada a unas reglas que, aunque igualitarias e impersonales por naturaleza, oprimen sin parecerlo a quienes no ejercen el control sobre los recursos económicos y políticos(...) haciendo jurídicos los conflictos sociales; los nuevos servicios jurídicos contribuyen involuntariamente, y a menudo inconscientemente, a la constitución y la reproducción de las clases subordinadas.

Sobre esta crisis del sistema jurídico, pueden verse dos textos muy distintos entre sí, pero pertinentes al objeto de este escrito: el primer capítulo del trabajo de Bonafé (1991) y el de Santos (1998 b). No obstante las diferencias, los dos trabajos se ocupan de analizar los elementos en los que se define una tensión estructural al interior de los conflictos jurídicos, de cara al derecho y al aparato jurisdiccional.

10 Por ejemplo, como lo muestra Wolkmer, la estrategia más recurrida para sacar los asuntos colectivos de la órbita de la legalidad sería vinculando entidades no judiciales al manejo de la conflictividad, como por ejemplo de la rama ejecutiva del Estado.

11 ARDILA, Édgar. Justicia comunitaria como realidad contemporánea: fundamentos para el análisis de las políticas públicas. Bogotá, 2003.

12 EBERHARD, Cristoph. Algunas implicaciones de los enfoques alternativos: el otro derecho 30. Bogotá: ILSA, 2004. 